\title{
Faster publication isn't always better
}

\author{
Valentin Fuster and Michael E Farkouh
}

With regards to health and safety, the public has both a right and need to know all information available. It is important, however, that studies provoking questions about a drug's safety, such as the New England Journal of Medicine (NEJM) paper on diabetes treatment with Avandia $^{\circledR}$ (SB Pharmaco Puerto Rico Inc., a subsidiary of GlaxoSmithKline), undergo thorough analysis. Otherwise, the public is done a disservice.

The NEJM meta-analysis of the cardiovascular safety profile of Avandia ${ }^{\circledR}$ seems a rushed and incomplete examination. The issue of cardiovascular safety would have been better dealt with through a much deeper prepublication scientific review, avoiding chaos amongst patients and unnecessary sensationalism. Of course, trials must be halted and data released immediately if there is overwhelming evidence of significant benefit or harm. In the case of this meta-analysis, which presented an analysis of studies that were never designed to be pooled, the small excess in cardiac events reported might or might not be attributed to Avandia ${ }^{\circledR}$. Classically, meta-analysis can only provide insights into the direction of treatment effects rather than the magnitude of such effects. Many have advocated scientific rigor similar to that seen in clinical trials be incorporated into metaanalysis design. This meta-analysis, therefore, should have started the discussion, not been the subject of disturbing headlines.

The NEJM paper suffers from several serious limitations, three of which deserve specific attention. First, the 42 trials included did not have the same protocol. Patients excluded in one trial could have been included in the next. Some trials compared Avandia ${ }^{\circledR}$ with placebo; others tested it against an active comparator. Furthermore, the trials were of different durations and tested different dosing regimens. Second, this analysis used published data only. This lack of first-hand source data means outcomes could not be verified, doublechecked or examined closely. Third, as admitted
So does

Avandia ${ }^{\circledR}$

increase the

risk of heart

attack or

cardiovascular-

related death?

The analysis

published in

NEJM does not

answer this

question.

$V$ Fuster is the Editorin-Chief of Nature

Clinical Practice

Cardiovascular

Medicine. ME Farkouh

is Associate Professor

of Medicine at the

Mount Sinai School of

Medicine, New York,

NY, USA.

Competing interests

V Fuster declared

associations with

GlaxoSmithKline. See

the article online for full

details of the relationship

ME Farkouh declared no

competing interests.

www.nature.com/clinicalpractice doi:10.1038/ncpcardio0942 in the meta-analysis, the conclusions are made on the basis of a small number of events "that could be affected by small changes in the classification of events". Many studies included were designed to assess end points other than cardiovascular disease.

Most physicians and research scientists know that this type of retrospective, pooling analysis is not the gold standard for answering safety questions or changing prescribing practices. A large, multi-center, randomized, double-blind trial, ADOPT, which examined 4,360 drug-naive, recently diagnosed diabetics found no difference in ischemic events with Avandia ${ }^{\circledR}$ compared with metformin, a drug with an established cardiovascular benefit. An interim analysis of the RECORD trial $(n=4,458)$ showed that there is no significant increase in the primary end point of cardiovascular-related hospitalization or death with Avandia ${ }^{\circledR}$. Furthermore for cardiovascular-related death alone, RECORD showed a point estimate of a $17 \%$ decrease in events with Avandia ${ }^{\circledR}$-the opposite of that demonstrated in the meta-analysis (odds ratio 1.64).

Does Avandia ${ }^{\circledR}$ increase the risk of heart attack or cardiovascular-related death? The analysis published in NEJM does not answer this question. Neither publication deadlines or congressional schedules should serve as the catalyst for releasing incomplete data; the public will not understand the difference between a limited retrospective meta-analysis and a well-designed prospective evaluation. The treatment of type-2 diabetes is already a daunting task. Unfortunately when patients, many of whom already do not comply with medication regimens, hear that a prescribed drug has harmful effects, it's hard for doctors to regain their trust. Faster and incomplete publication isn't always better, particularly when the public and political implications may be huge, as demonstrated after publication of the NEJM report. 